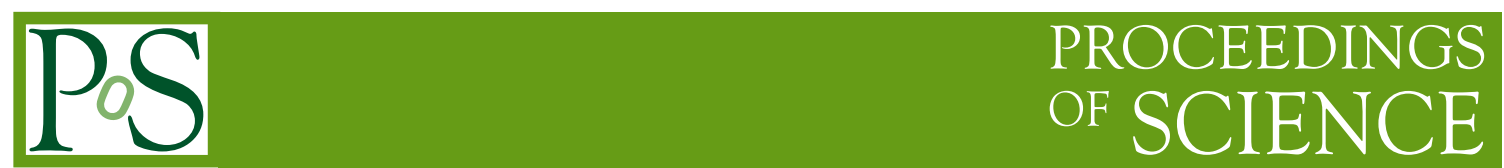

\title{
Cosmic rays: direct measurements
}

\author{
Paolo Maestro \\ Department of Physical Sciences, Earth and Environment \\ University of Siena, via Roma 56, 53100 Siena (Italy) \\ E-mail: paolo.maestro@pi.infn.it
}

This paper is based on the rapporteur talk given at the $34^{\text {th }}$ International Cosmic Ray Conference (ICRC), on August $6^{\text {th }}, 2015$. The purpose of the talk and paper is to provide a summary of the most recent results from balloon-borne and space-based experiments presented at the conference, and give an overview of the future missions and developments foreseen in this field.

The 34th International Cosmic Ray Conference,

30 July- 6 August, 2015

The Hague, The Netherlands 


\section{Introduction}

I will report on the papers concerning the direct measurements of cosmic rays (CRs) presented at the $34^{\text {th }}$ ICRC, held in The Hague from July $30^{\text {th }}$ to August $6^{\text {th }}, 2015$. I identified about 110 contributions relevant for this topic, mainly presented in the CR sessions (CR-EXperiments (45), CR-INstrumentation (23), CR-THeory (25)) and Dark Matter sessions (DM-IN (10), DM-EX (2), DM-TH (4)). Since it would be impossible to review all of them, I tried to highlight the ones I consider of interest for the broad CR community and which in my opinion better represent the status of the art in the field. In spite of my effort to be as much unbiased as possible, I realize that my judgement in this matter could be imperfect and the choice of topics inevitably affected by my research interests and experimentalist's point of view, and therefore I apologize for those contributions I omitted to mention.

A wealth of new results obtained by balloon-borne and space-based experiments have been presented at the conference, covering several observation targets from nuclei and isotopes, to electrons and positrons, to antiprotons, measured over six decades in energy from few $\mathrm{MeV}$ up to some TeV. The measurements from the AMS-02 and PAMELA spectrometers are characterized by a precision never reached before in CR history; they allowed to discover features in the CR energy spectra that compel a revision of the simple idea that CR fluxes are described by smooth single power-laws in energy, and prompted an intense theoretical activity to interpret the results. Among the most interesting outcomes, the first measurement of the CR spectra outside the heliosphere (Voyager 1), the high-statistics measurement of the abundances of nuclei heavier than iron (SuperTIGER), and the first detection of a primary CR clock (ACE-CRIS) are certainly worth considering. Moreover upcoming calorimetric missions (NUCLEON, CALET, DAMPE, ISS-CREAM) are foreseen to collect in the next few years unprecedented statistics of CRs even at higher energies, in the multi-TeV range and finally approaching the knee. All these advances indicate that we have entered a new era of precision measurements of CRs, which can contribute to shed light on several fundamental questions in CR physics, still open a century after their discovery, like the mechanism of acceleration of galactic cosmic rays (GCRs), the nature and composition of their sources, the $\mathrm{CR}$ propagation in the interstellar medium (ISM).

This paper is organized as the rapporteur talk, i.e. according to observation targets. In section 2 the most recent measurements of proton and helium are discussed, followed by results on heavier nuclei in section 3 . Section 4 deals with electrons, while measurements of the antimatter component $\left(\mathrm{e}^{+}, \bar{p}\right)$ of CRs are covered in section 5. In each section, I briefly summarize the main theoretical models which have been proposed to interpret the results, though a complete discussion of this subject is beyond the scope of the paper. A review about the upcoming missions and proposals of new instrumentation and experiments is presented in section 6. My conclusions are drawn in section 7

\section{Proton and helium}

Direct measurements of the energy and charge of CR nuclei are performed by space-based or balloon-borne instruments exploiting various detection techniques, depending on the energy region of interest. Stack of solid-state detectors are used up to hundreds of MeV/n, Cerenkov detectors 
up to few $\mathrm{GeV} / \mathrm{n}$, magnetic spectrometers in the energy range from few $\mathrm{GeV} / \mathrm{n}$ up to few $\mathrm{TeV} / \mathrm{n}$, nuclear emulsions, calorimeters, transition radiation detectors up to hundreds of TeV. Protons and helium nuclei are the most abundant components of cosmic radiation. Precise measurements of their fluxes at high energies can provide new insights into the nature and origin of CRs, while low-energy data are important for studying the CR interactions with ISM, heliosphere and magnetosphere.

\subsection{High-energy region}

The highest-energy data for proton and helium were obtained in the 1990's by balloon experiments using nuclear emulsions (RUNJOB, JACEE), but they are affected by large errors and inconsistent with each other [1,2]. Measurements from JACEE suggested a steeper spectrum for $p$ with respect to He, but subsequent data obtained by RUNJOB did not confirm this result and were consistent with a similar spectral index for the two lightest elements. This controversy was solved by the balloon-borne experiment CREAM that collected the largest statistics of CR protons and helium nuclei above $100 \mathrm{TeV}$, showing unequivocally that $p$ and $\mathrm{He}$ spectra in the multi-TeV energy region are harder than the energy spectra at lower energies $(<100 \mathrm{GeV} / \mathrm{n})$ and described by power-laws $E^{\gamma}$ with different spectral indices $\left(\gamma_{p}=-2.66 \pm 0.02\right.$ and $\gamma_{H e}=-2.58 \pm 0.02$ in the particle energy range 2.5-250 TeV), with the proton softer than the He spectrum [3]. Then the only way to reconcile the higher and lower energy data is assuming a hardening of the spectra in the hundred $\mathrm{GeV}$ region. This was first observed by the magnetic spectrometer PAMELA that reported spectral shapes for $p$ and He clearly inconsistent with single power-laws. PAMELA $p$ and He data exhibit an abrupt spectral hardening occurring between 230 and $240 \mathrm{GV}$, with a change $\Delta \gamma$ of the spectral index $\sim 0.2$ for $p$ and 0.3 for He, respectively [4].

At this ICRC, AMS presented its final results on $p$ and He confirming the break and providing a precise measurement of the transition to harder spectra. AMS-02 is a precision, multipurpose spectrometer launched in 2011 and installed on the International Space Station (ISS) [5]. The core of the instrument is a $0.14 \mathrm{~T}$ permanent magnet instrumented with nine layers of Si microstrip detectors, six of which are within the bore of the magnet. The tracker accurately determines the trajectory of charged particles in the magnetic field and measures the particle rigidity $R=P / Z(P$ is the momentum and $Z$ the charge of the particle) from its curvature. The spatial resolution in each layer is $10(7.5) \mu \mathrm{m}$ for $p(\mathrm{He})$ in the bending direction, and the maximum detectable rigidity (MDR), where the error of the measured rigidity becomes $100 \%$, is 2 TV for $p$ and 3.2 TV for $\mathrm{He}$, respectively. The tracker can also determine the absolute value of $Z$ by multiple $\mathrm{dE} / \mathrm{dx}$ with a charge resolution $\Delta Z \approx 0.05$ for $|Z|=1$ and 0.07 for $|Z|=2$. In addition to the tracker, independent and redundant charge and energy measurements are provided by Time of Flight counters (TOF), a Ring Image Cerenkov Counter (RICH), and an Electromagnetic Calorimeter (ECAL). A Transition Radiation Detector (TRD) is used to separate electrons and from protons, while a veto system (ACC) rejects with an efficiency of 0.99999 CRs which enter the inner tracker from the side.

In four years of data taking AMS- 02 collected about $68 \times 10^{9} \mathrm{CR}$ events, more than the whole statistics of all the previous balloon-borne and space-based experiments. A final selection of $300 \times 10^{6}$ protons and $50 \times 10^{6} \mathrm{He}$ nuclei was used to measure the differential fluxes as a function of rigidity, in the range between $1 \mathrm{GV}$ and $1.8 \mathrm{TV}$ for $p$ [6,7], and from $1.9 \mathrm{GV}$ to $3 \mathrm{TV}$ for He [8]. Such a huge statistics allowed a very accurate study of the systematic uncertainties affecting the flux 


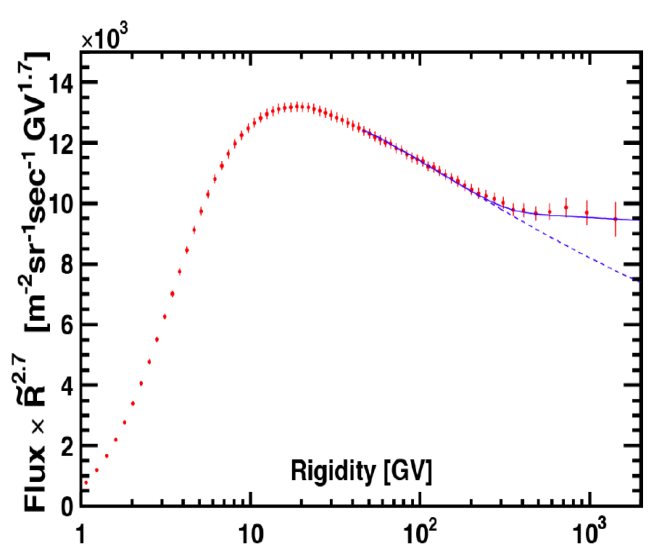

(a)

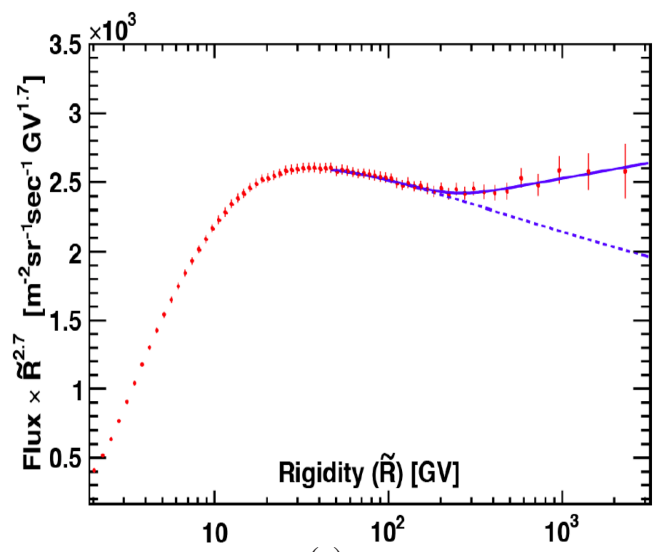

(c)

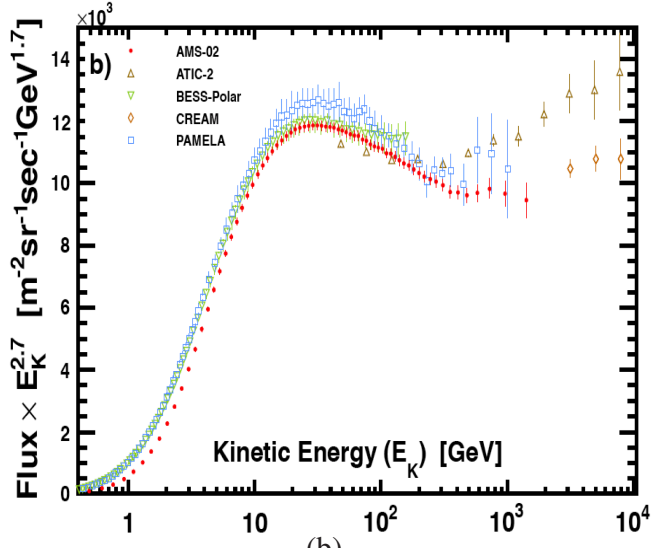

(b)

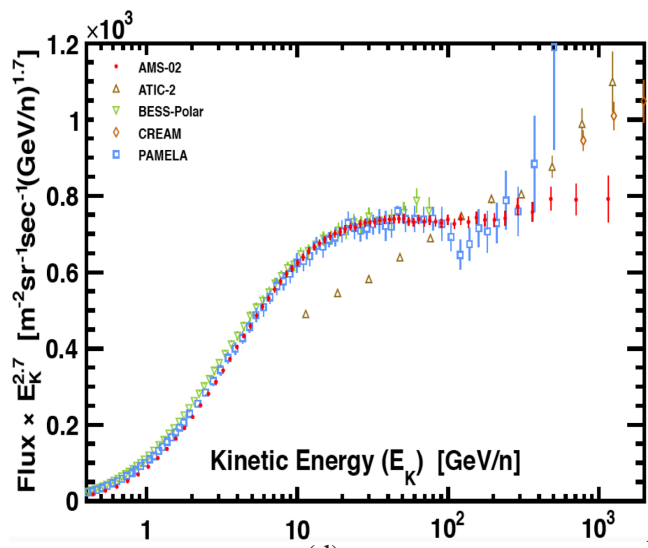

(d)

Figure 1: AMS (a) $p$ and (c) He fluxes multiplied by $R^{2.7}$ as a function of rigidity $R$. Curves represent the fit of Eq. 2.1 (solid line) and a single power-law (dotted line) to the data. AMS (b) $p$ and (d) He fluxes multiplied by $E_{k}^{2.7}$ as a function of kinetic energy per nucleon $E_{k}$, compared with the most recent results from spectrometers (BESS, PAMELA) and calorimetric experiments (ATIC, CREAM) [6, 8].

measurements, comparable to the sophisticated analyses of collider experiments. The total systematic error includes uncertainties stemming from several sources, like the trigger efficiency, the acceptance, the background contamination, the geomagnetic cutoff factor, the event selection, the unfolding, the rigidity resolution function, and the absolute rigidity scale. Systematic errors dominate in whole rigidity range, the statistical error being less than $1 \%$. The total $p$ flux error is $4 \%$ at $1 \mathrm{GV},<2 \%$ between 2 and $350 \mathrm{GV}$, and $6 \%$ above $1.1 \mathrm{TV}$. The AMS $p$ and He spectra are shown in Figs. 1(a) and 1(c) respectively. They clearly show a deviation with respect to a single power-law behaviour $R^{\gamma}$ around $300 \mathrm{GV}$. The data above $45 \mathrm{GV}$ can be fitted to a double power-law function

$$
\Phi=C\left(\frac{R}{45 G V}\right)^{\gamma}\left[1+\left(\frac{R}{R_{0}}\right)^{\frac{\Delta \gamma}{s}}\right]^{s}
$$

with a smooth transition from a spectral index $\gamma$ below the transition rigidity $R_{0}$ to $\gamma+\Delta \gamma$ above. The fitted spectral hardening is $\Delta \gamma=0.133_{-0.021}^{+0.032}(\mathrm{fit})_{-0.030}^{+0.046}(\mathrm{sys}) \pm 0.005$ (sol) 1 for $p$ occurring at $R_{0}=$

\footnotetext{
${ }^{1}$ The first error (fit) stems from statistical and uncorrelated systematic errors of the flux. The second (sys) is the
} 
$336_{-44}^{+68}(\mathrm{fit})_{-28}^{+66}$ (sys) \pm 1 (sol) GV. For He, $\Delta \gamma=0.119_{-0.010}^{+0.013}(\mathrm{fit})_{-0.028}^{+0.033}$ (sys) and $R_{0}=245_{-31}^{+35}(\mathrm{fit})_{-30}^{+33}$ (sys) GV. Then $p$ and He spectra have very similar rigidity dependence, but the He spectral index is different from that of $p: \gamma_{H e}=-2.780 \pm 0.005$ (fit) \pm 0.001 (sys) vs. $\gamma_{p}=-2.849 \pm 0.002$ (fit) $\pm_{-0.003}^{+0.004}$ (sys) ${ }_{-0.003}^{+0.004}(\mathrm{sol})$, respectively. Moreover, by studying the detailed variation of $\gamma$ with rigidity, it turns out that the spectral index progressively hardens above $100 \mathrm{GV}$ for both species, but still $p$ is softer than He. The $p / \mathrm{He}$ flux ratio can be fitted to a single-power law in rigidity above $45 \mathrm{GV}$ with a constant spectral index $-0.077 \pm 0.002$ (stat) \pm 0.007 (sys) [8].

In Figs. 1(b) and 1(d) the AMS results are compared with the most recent measurements by magnetic spectrometers (PAMELA [4], BESS-Polar II [9]) and balloon-borne calorimetric experiments (ATIC [10], CREAM [3]). In [11], it was pointed out that PAMELA and AMS results agree very well: in the energy region not affected by solar modulation, i.e. at rigidities $>30 \mathrm{GV}$, the average ratio between the fluxes measured by the two experiments is 0.988 for $p$ and 1.036 for He. It is the first time in the history of CRs that the absolute fluxes reported by different experiments differ at the level of percent. Another remark is that, unless exploiting the transition radiation detector to extend the range of energy measurement for $Z \geq 2$ nuclei, as proposed in [12], AMS cannot push the measurement of $p$ and He spectra beyond its MDR, which is few TeV/n. Then next accurate measurements of $p$ and He fluxes over the spectral break and extending up to hundreds of $\mathrm{TeV}$ might come from future calorimetric experiments (see section 6).

These new results force us to revise the standard model of GCRs and find theoretical explanations for the origin of the spectral hardening. The standard paradigm is based on diffusive shock acceleration (DSA) of charged particles in supernova remnants (SNRs), with an injection spectrum described by a power-law in energy $E^{\alpha}$, followed by CR propagation in the ISM, with an escape time from the Galaxy proportional to $E^{\delta}$. It predicts that the spectra of primary CRs observed at Earth follow a power-law $E^{\gamma}$ with spectral index $\gamma=\alpha+\delta \approx-2.7$, i.e. steeper than the injection spectra. Several theoretical break models have been proposed to explain the observed deviation from a single power-law behaviour; they can be grouped essentially in three main classes [13]. In the first class, the hardening is interpreted as a feature due to the acceleration mechanism of CRs at the source. For instance, it could be the effect of distributed acceleration by multiple SNRs in OB associations or Superbubbles [14, 15], or an effect of reacceleration of CRs by weak shocks in the Galaxy [16], or alternatively it could represent the spectral concavity caused by the interactions of accelerated CRs with the accelerating shock, as foreseen in non-liner DSA models [17, 18]. In the second class of theoretical break models, the spectral hardening could originate from subtle effects of CR propagation, like an inhomogeneous diffusion, characterized by a different energy dependence in different region of the Galaxy [19], or a non-linear coupling of CRs with the diffusion coefficient, typical of models in which the transport of GCRs is regulated by self-generated waves [20,21]. Finally, the spectral hardening could be due to the effect of local young sources [22-24], or to a mixture of fluxes accelerated by sources with different properties (e.g. few old local SNRs vs. an ensemble of young and distant SNRs) contributing in different energy region of the spectra [25]. Different ideas were also explored to explain the softer $p$ spectrum with respect to $\mathrm{He}$ and heavier nuclei, e.g. different astrophysical sources for $p$ and $\mathrm{He}$ [26], an injection in the acceleration cycle more efficient for He than for $p$ [27], variable He and $p$ con-

error from the remaining systematic errors; the third (sol) is due to the variation of the solar potential [7]. 
centrations in SNR environments [28,29], different spallation rates for $p$ and heavier nuclei during propagation time [30].
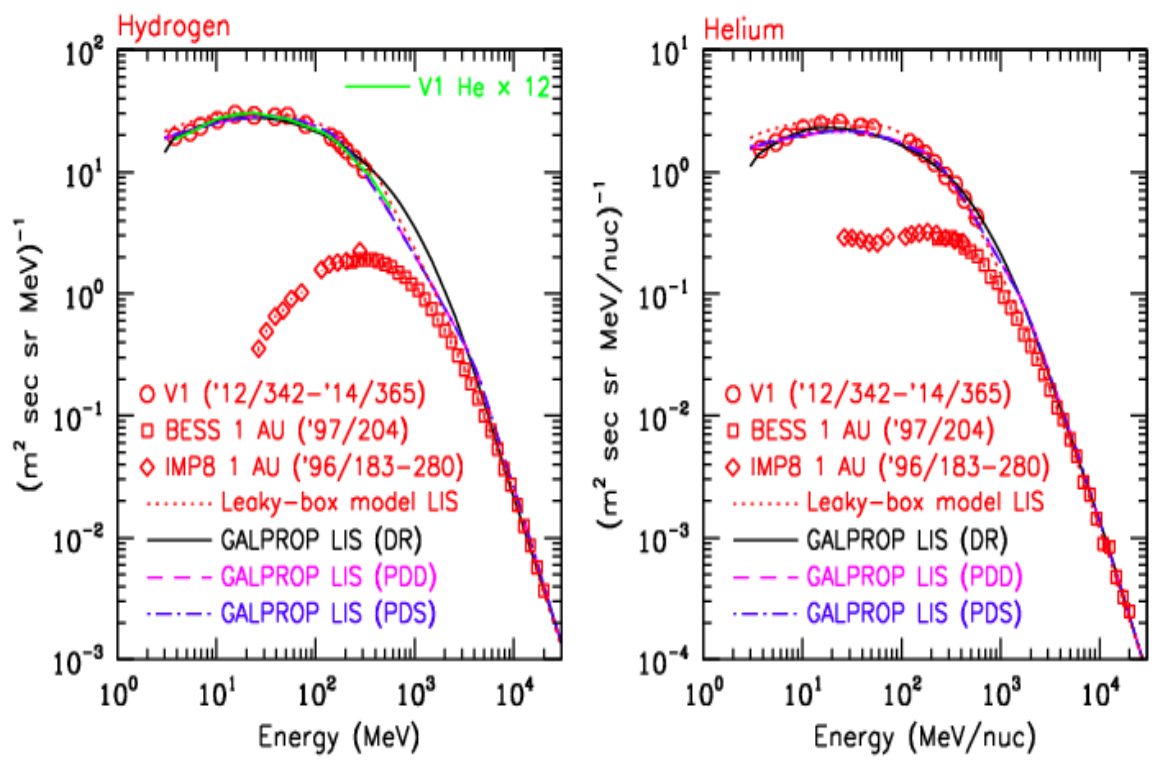

Figure 2: Energy spectra of $\mathrm{H}$ (left) and He (right) from Voyager 1, compared with solar-modulated spectra at 1 AU from BESS and IMP8, and predictions from different propagation models [34].

\subsection{Low-energy region $(<10 \mathrm{GeV})$}

The large statistics of low-energy proton data collected by PAMELA and AMS is extremely important to enhance our understanding of solar modulation and geomagnetic effects on CRs. The first can be investigated by studying the time evolution of proton flux over several years. At the ICRC, AMS reported the detailed time variation of the $p$ flux from 1 to $10 \mathrm{GV}$ over the period 2011-2013, showing not only the expected overall flux decrease with increasing solar activity until its maximum in 2014, but also periodic monthly variations of the flux related to strong solar events, i.e. Coronal Mass Ejections and Forbush decreases [31]. New accurate measurements of undercutoff proton fluxes from $70 \mathrm{MeV}$ up to few $\mathrm{GeV}$ at low Earth orbit were presented by PAMELA. Protons were classified into geomagnetically trapped and re-entrant albedo on the basis of particle tracing techniques in a wide geomagnetic region, allowing more accurate view of atmospheric and geomagnetic effects on the $\mathrm{CR}$ transport, and a better characterization of the radiation environment near the Earth [32].

The spectra of $\mathrm{H}$ and $\mathrm{He}$ were measured for the first time in their unmodulated state by the Cosmic Ray Subsystem (CRS) experiment on Voyager 1 (V1). The V1 space probe left the heliosphere on August 2012 and entered the denser and colder environment of the interstellar cloud surrounding the solar system. After crossing the heliopause at a radial distance of $121.6 \mathrm{AU}$, the intensity of GCRs reached the highest level observed over the 35 years of V1 journey and remained constant for the next two years. At the same time a depletion of heliospheric ions, which escaped 
into the interstellar space, was observed. It turned out that the heliosphere operates as a shield that excludes more than $75 \%$ of the GCRs with energy $>70 \mathrm{MeV}$ [33]. Then the data collected in the ISM by the CRS solid state detector telescopes allowed to reveal the low energy part of the interstellar CR spectra from $\mathrm{H}$ to $\mathrm{Ni}$, i.e. not modulated by the time-dependent solar wind. Very similar spectra for $\mathrm{H}$ and $\mathrm{He}$ have been measured in the range 3-346 MeV/n, as shown in Fig. 2. The $\mathrm{H} / \mathrm{He}$ ratio is $\sim 12$ independent of energy. Both the $\mathrm{H}$ and $\mathrm{He}$ spectra exhibit a broad peak at the same energy per nucleon, implying that thay are not affected by solar modulation and supporting the idea that V1 is really travelling in the local ISM. The roll over at low energies from a power-law at high energies is likely a consequence primarily of ionization energy losses and suggests that $\mathrm{V} 1$ is not in the nearby of a recent source of GCRs [34].

New isotopic measurements of $\mathrm{H}$ and He were also presented by PAMELA and BESS-Polar. ${ }^{2} \mathrm{H}$ and ${ }^{3} \mathrm{He}$ are mostly secondary particles produced by the interaction of primary ${ }^{1} \mathrm{H}$ and ${ }^{4} \mathrm{He} \mathrm{CRs}$ with the ISM during propagation. Then the secondary-to-primary ratios ${ }^{2} \mathrm{H} /{ }^{1} \mathrm{H}$ and ${ }^{3} \mathrm{He} /{ }^{4} \mathrm{He}$ provide important information on propagation history of CRs in the ISM, and they are complementary to $\mathrm{B} / \mathrm{C}$ measurements in constraining propagation models. Combining a tracking system based on a $0.8 \mathrm{~T}$ superconducting magnet and a JET-type drift chamber, with a time-of-flight hodoscope and an aerogel Cherenkov counter, the balloon-borne BESS-Polar II instrument measured these isotopes with an excellent mass separation up to $1.5 \mathrm{GeV} / \mathrm{n}$. The isotope ratios from data taken in a 24.5-day Antarctic flight in 2007, during a solar minimum, are shown in Fig. 3. These results have unprecedented precision and allow to discriminate between different propagation models [35]. While the $\mathrm{H}$ isotopes ratio is consistent with previously published PAMELA data [36], taken during the same solar minimum, there is an evident disagreement as far as ${ }^{3} \mathrm{He} /{ }^{4} \mathrm{He}$ ratio is concerned. PAMELA presented new preliminary measurements of the isotopic composition [37], based on a more refined analysis procedure than the previous work [36] and extending to higher energies, but a comparison with the new BESS-Polar data was not yet shown.

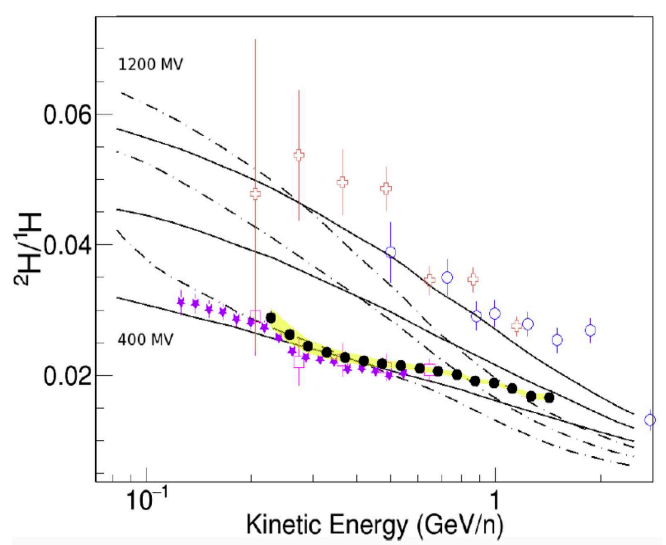

(a)

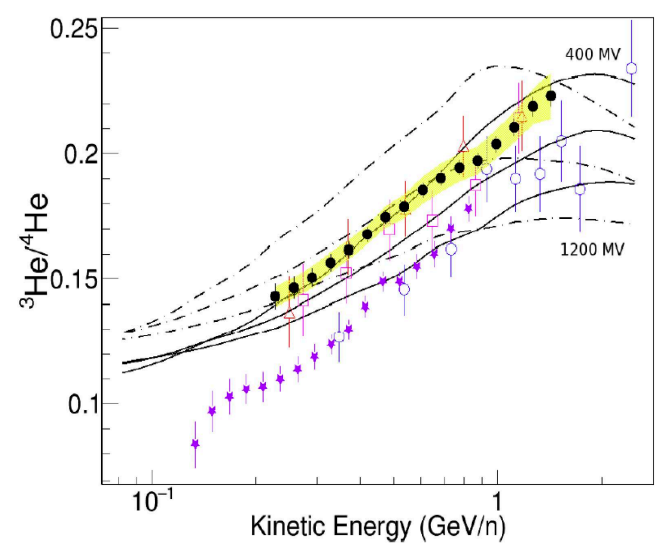

(b)

Figure 3: Ratios of (a) ${ }^{2} \mathrm{H} /{ }^{1} \mathrm{H}$ and (b) ${ }^{3} \mathrm{He} /{ }^{4} \mathrm{He}$ as measured by BESS-Polar II (filled circles), compared with previous data from IMAX-92 (open circles), BESS-93 (open squares), AMS-01 (open triangles) and PAMELA (filled stars). The lines represent "Plain Diffusion" (solid) and "Reacceleration" (dashed) models, calculated with GALPROP for different solar modulation parameters [35]. 


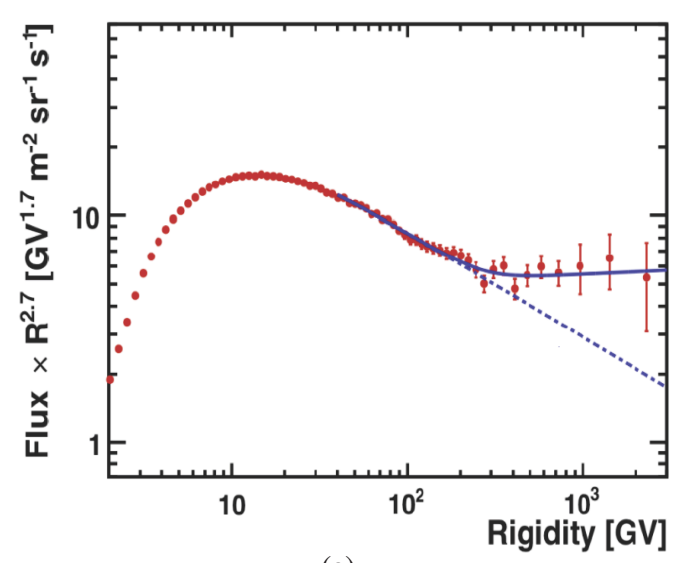

(a)

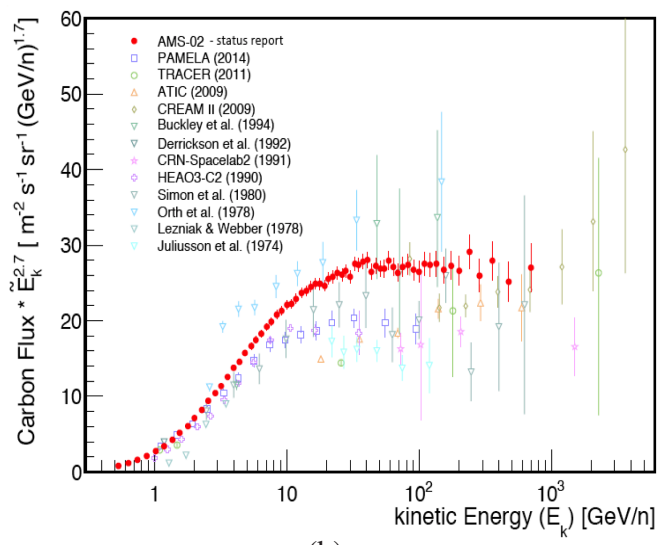

(b)

Figure 4: (a) The AMS Li flux multiplied by $R^{2.7}$ as a function of rigidity $R$ [41]. (b) The AMS C flux multiplied by $E_{k}^{2.7}$ as a function of kinetic energy per nucleon $E_{k}$, compared with earlier results [42].

\section{Nuclei with $\mathrm{Z}>2$}

\subsection{Light nuclei}

In the last decade the balloon-borne experiments CREAM [38] and TRACER [39] measured the energy spectra of the most abundant primary heavy nuclei $(\mathrm{C}, \mathrm{O}, \mathrm{Ne}, \mathrm{Mg}, \mathrm{Si}, \mathrm{Fe})$ up to energy of $\sim 10^{14} \mathrm{eV}$ per particle. All the spectra are well fitted to single power-laws in energy with a remarkably similar spectral index $\gamma \approx-2.65$ which well agrees with that of $\mathrm{He}$ in the multi-TeV region, implying that all these elements likely have the same origin and share the same acceleration and propagation processes. However, by fitting simultaneously the normalized energy spectra to a broken-power law, CREAM reported also a hint of a spectral hardening above $\sim 200 \mathrm{GeV} / \mathrm{n}$ [40], similar to that observed for $p$ and He. At the moment, the statistical and systematic uncertainties of these measurements significantly hinder a conclusive interpretation of the data. Further accurate observations are needed to extend with high statistics the present data to higher energies, on the one hand, and measure accurately a possible curvature of the spectrum and the position of the spectral break-point for individual nuclear species, on the other hand. While only future calorimetric experiments will be able to address the first task, AMS has the unique opportunity to accurately investigate the region between $100 \mathrm{GeV} / \mathrm{n}$ and few $\mathrm{TeV} / \mathrm{n}$ to search for possible spectral features. So far, AMS reported only preliminary measurements of the energy spectra of lithium and carbon. Lithium, like Be and B, has a secondary origin, i.e. it is produced by the spallation of heavier CR nuclei with the ISM during their propagation. Measurements of these light nuclei spectra, as well as of secondary-to-primary abundance ratios, allow to probe galactic propagation models and constrain their parameters. Since $\mathrm{Li}$ accounts for only about $0.1 \%$ of CRs, its measurement is really challenging and few data from old balloon experiments were available so far. Thanks to its large geometrical factor and exposure, AMS measured about $1.5 \times 10^{6} \mathrm{Li}$ events. The independent charge identification provided by TOF and tracker allowed to select a pure sample of $\mathrm{Li}$ events, with small contamination of heavier CR nuclei interacting in the upper part of the detector and producing lithium. The differential Li flux has been measured between $2 \mathrm{GV}$ and $3 \mathrm{TV}$ [41], showing an unexpected hardening above $200 \mathrm{GV}$ (Fig. 4(a) , the same rigidity region as for $p$ and 


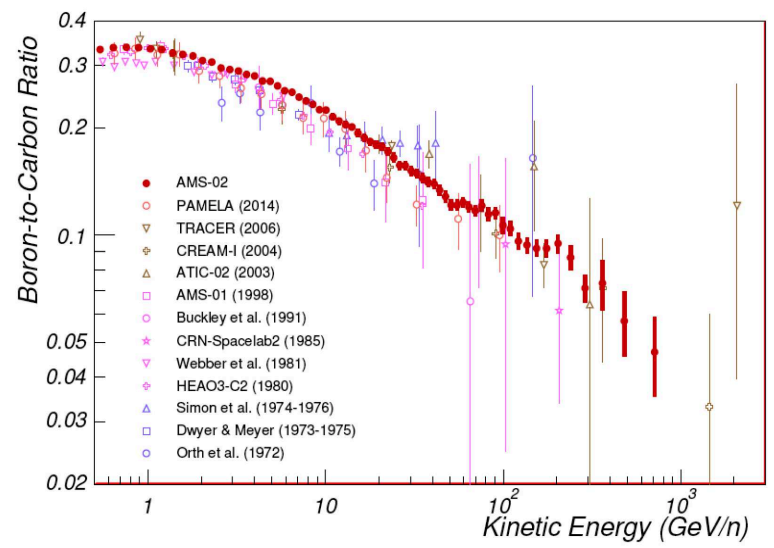

Figure 5: New AMS B/C ratio compared with a compilation of earlier measurements [48].

He. Surprisingly such a feature is not observed in the AMS carbon spectrum [42], which above 45 GV seems to be compatible with a single power-law (Fig. 4(b)]. Nevertheless, it has been pointed out that, unlike the $p$ and He data, these AMS measurements are preliminary, since the statistical errors are still dominant over systematics above $100 \mathrm{GV}$; more data need to be collected to accurately determine the shape of these spectra at high energies. It is also worth noting the significant discrepancy between the AMS and PAMELA carbon fluxes, which clashes with the general good agreement observed between their $p$ and $\mathrm{He}$ data. New preliminary results on the $\mathrm{Li}$ and $\mathrm{Be}$ fluxes from 1 to $100 \mathrm{GV}$ [43] and their isotopic composition up to $1.2 \mathrm{GeV} / \mathrm{n}$ [44] were also presented at the ICRC by PAMELA, but these analyses are still in an early stage.

Information about the diffusion of CRs in the Galaxy can be inferred from the measurement of the secondary-to-primary abundances (e.g. B/C, sub-Fe/Fe ratios), which allow to estimate the average amount of material that CRs typically traverse between injection and observation. The escape pathlength $\lambda$ of CRs from the Galaxy is known to decrease as the particle rigidity increases, following a power-law $\lambda \propto R^{\delta}$, where $\delta$ is the diffusion coefficient index. This is a key parameter and its accurate measurement is crucial to derive the injection spectrum at the source by correcting the observed spectral shape for the energy dependence of the propagation term. Pre-AMS B/C data favor a value of $\delta \approx-0.6$ at energies of few $\mathrm{GeV} / \mathrm{n}$, while high-energy data seems to flatten to $\delta \approx-0.4$. However measurements by balloon-experiments (CREAM [45], ATIC [47], TRACER [46]) between $10^{2}$ and $10^{3} \mathrm{GeV} / \mathrm{n}$, suffer from statistical limitations and large systematic errors stemming from the corrections for nuclei produced by CR interaction with the residual atmosphere, and do not allow to tightly constrain the value of $\delta$ and discriminate among different propagation models. The new AMS data (Fig. 5), based on $7 \times 10^{6} \mathrm{C}$ and $2 \times 10^{6} \mathrm{~B}$ events, have unprecedented precision and accurately determine the spectral shape of the $\mathrm{B} / \mathrm{C}$ ratio up to about $700 \mathrm{GeV} / \mathrm{n}$ [48]. Given this high level of precision, the theoretical uncertainties affecting the models become the major limitation for the interpretation of data. In [49], it is shown that the lack of information about a possible injection of $\mathrm{B}$ nuclei at the sources and the errors on the nuclear cross sections seriously limit the extraction of $\mathrm{CR}$ diffusion parameters from $\mathrm{B} / \mathrm{C}$ data. In [50], the transport parameter space is scanned to find solutions for the propagation equations fitting simultaneously pre-AMS $\mathrm{B} / \mathrm{C}$ and other CR data. A large number of degenerated solutions were found which cannot be even completely resolved by further constraints from AMS data, but might be only reduced with a 


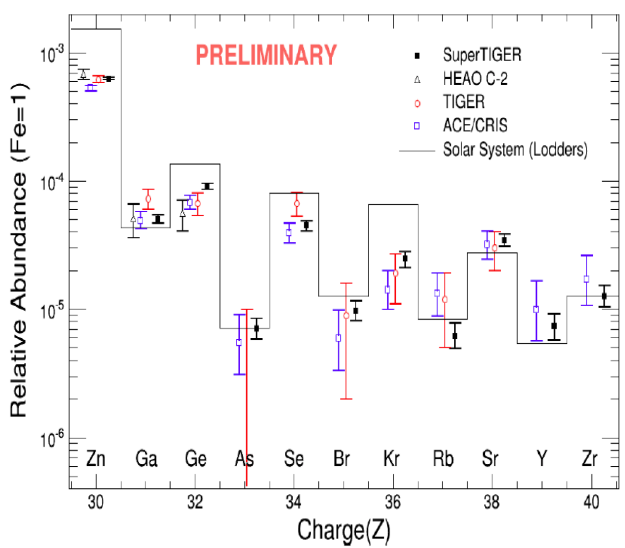

(a)

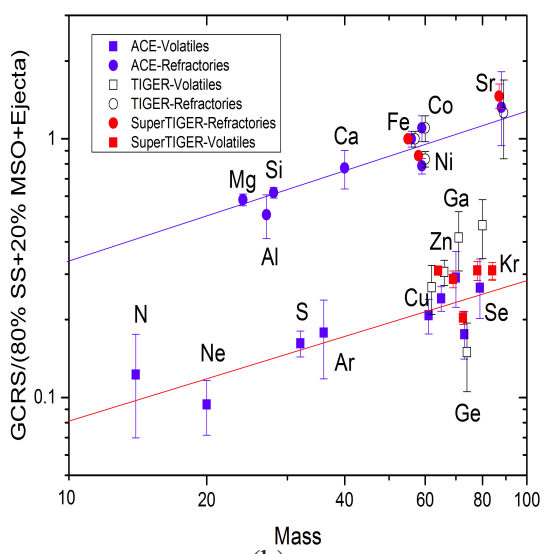

(b)

Figure 6: (a) Comparison of SuperTIGER Top-of-Atmosphere abundances with TIGER data and space abundances from ACE-CRIS, and HEAO. (b) GCR source abundances compared to a mixture of $80 \%$ solar system and 20\% from massive star outflow as a function of the nuclear mass. Preliminary SuperTIGER data (the errors are only statistical) are compared with previous results from ACE-CRIS and TIGER [56].

multi-messager approach.

Accurate measurements of $\mathrm{B} / \mathrm{C}$ ratio at $\mathrm{TV}$ rigidities are needed to test the spectral break models. It has been pointed out that an error $<10 \%$ on the $\mathrm{B} / \mathrm{C}$ ratio at $1 \mathrm{TeV} / \mathrm{n}$ would allow to discriminate between different classes of models [50]. AMS can reach this goal by collecting more data in the next years, since its preliminary result is still dominated by the statistical uncertainties above 60 GV. A propagation induced break would imply secondary spectra with a more pronounced break than primary ones, while no feature are expected in secondaries/primaries ratio if the break origin is in the source [13]. The significant spectral break observed by AMS for Li seems to point to the first scenario, but more data on other nuclei are needed to clarify the picture. However, in [51], the authors observe that $\mathrm{B} / \mathrm{C}$ ratio above $100 \mathrm{GeV} / \mathrm{n}$ is somewhat higher than the prediction of a non-linear CR Galactic transport model used to explain the spectral break of $p$ and He fluxes. That could indicate a contribution of secondary production at the source that may become significant at $\mathrm{TeV} / \mathrm{n}$ scale, thus further complicating the possibility to disentangle source and propagation effects.

\subsection{Iron and ultra-heavy nuclei}

Elements above $\mathrm{Fe}$ are extremely rare compared to light elements, but they can provide unique information about the site of acceleration of GCRs. Measurements of the abundances of elements from $Z=30$ to $Z=38$ by the Trans-Iron Galactic Element Recorder (TIGER) [52] and the ratios of ${ }^{22} \mathrm{Ne} /{ }^{20} \mathrm{Ne},{ }^{58} \mathrm{Fe} /{ }^{56} \mathrm{Fe}$, and $\mathrm{C} / \mathrm{O}$ [53] by the Cosmic Ray Isotope Spectrometer (CRIS) onboard the Advanced Composition Explorer (ACE), support the idea of OB associations as CR acceleration sites. Since supernovae ( $\mathrm{SN}$ ) explode preferentially in groups of massive stars, named OB associations, then DSA would occur in an ISM of solar-system composition enriched in freshly synthesized material from previous SN or Wolf-Rayet star ejecta. New data confirming this scenario have been presented by the SuperTIGER collaboration. Featuring a $8.3 \mathrm{~m}^{2} \mathrm{sr}$ acceptance, this balloon-borne experiment measured the abundances of ultra-heavy GCRs (UHGCRs) from Z $=30$ up to $Z=40$ with unprecedented statistics in a 55-day flight over Antarctica in 2012-13 [54]. The SuperTIGER instrument is made of two identical modules, each one consisting of two scintillat- 
ing fiber hodoscopes for particle tracking, three scintillators and two Cerenkov detectors, equipped with acrylic and aerogel radiators respectively, to measure the particle charge and velocity in the range $0.8-10 \mathrm{GeV} / \mathrm{n}$. The instrument is capable of separating each individual species from $\mathrm{Ne}$ to $\mathrm{Ba}$ with excellent resolution (0.16 charge units for $\mathrm{Fe}$ ) [55]. Observed elemental abundances of UHGCRs are corrected first for the residual atmospheric overburden (Fig. 6(a) and then for the effects of fragmentation in the ISM to derive the source abundances. The ratios of these calculated GCR source abundances to a mixture of $\sim 20 \%$ ejecta of massive stars (including Wolf-Rayet stars and core-collapse supernovae) mixed with $\sim 80 \%$ material of solar system composition, are plotted as a function of the elemental atomic mass in Fig. 6(b) By assuming this source mixture, a clear separation of refractory and volatile elements and a better ordering by mass is obtained with respect to a pure solar system composition, indicating that the refractory elements (found in interstellar dust grains) are more effectively accelerated than volatile ones (present in interstellar gas) [56]. These preliminary measurements are consistent with previous observations by TIGER and ACE-CRIS (performed in a lower energy range, 150-600 MeV/n), and provide an improved test of the origin of GCR in OB associations and the volatility model of GCR acceleration.

SuperTIGER also presented a very preliminary measurement of $(\mathrm{Sc}+\mathrm{Ti}+\mathrm{V}) / \mathrm{Fe}$ ratio in two energy bins defined by the thresholds of the two Cerenkov detectors [57]. Future plans are to calibrate their velocity scale and study the energy dependence of this secondary-to-primary ratio with finer resolution.

Another proof supporting the OB associations model for GCR origin, comes from the first measurement of a primary cosmic-ray clock, reported by ACE-CRIS [58]. CRIS can provide an excellent elemental and isotopic separation by measuring the $\mathrm{dE} / \mathrm{dx}$ and total energy of CRs stopping in a stack of silicon detectors. Based on 16.8 years of collected data, CRIS was able to detect 15 well-resolved $\left(\sim 0.24\right.$ mass resolution) events of the rare radioactive ${ }^{60} \mathrm{Fe}$ isotope in a set of $2.95 \times 10^{5}{ }^{56} \mathrm{Fe}$ nuclei, measured in the $240-470 \mathrm{MeV} / \mathrm{n}$ energy interval. A detailed analysis demonstrated that at most 2 out of $15^{60} \mathrm{Fe}$ could have been produced by interstellar fragmentation of Ni isotopes or could be spill-over from ${ }^{58} \mathrm{Fe}$. Then the observed ${ }^{60} \mathrm{Fe}$ are almost all primary nuclei. Since ${ }^{60} \mathrm{Fe}$ decays by $\beta^{-}$with a half-life of $2.62 \mathrm{Myr}$, the fact that we observe this radioisotope near Earth implies that the time $T$ elapsing between nucleosynthesis and CR acceleration is $\leq 10$ Myr. Moreover, a lower limit of $10^{5} \mathrm{yr}$ is inferred for $T$ from the lack of ${ }^{59} \mathrm{Ni}$ in CRs, as previosly reported by CRIS [59]. Combining this two limits on $T$ implies that CRs are not accelerated by the same $\mathrm{SN}$ in which they are synthesized, and then CR acceleration must take place in regions, like OB associations, where at least two nearby supernovae occur within a few Myr.

\section{Electrons}

The overall electron component $\left(e^{+}+e^{-}\right)$accounts for about $1 \%$ of CRs. Secondary electrons and positrons are produced in interactions of CR nuclei with the ISM, through the $\pi^{ \pm} \rightarrow \mu^{ \pm} \rightarrow e^{ \pm}$ decay chains. Since approximately equal amounts of $e^{-}$and $e^{+}$are expected from such processes, the observed overabundance of $e^{-}$over $e^{+}$in CRs indicates that most of electrons have a primary origin. Observations of $\mathrm{X}$-ray and $\mathrm{TeV}$ gamma-ray emission from SNRs provide clear evidence for the acceleration of electrons in SNR shock up to energies of about $100 \mathrm{TeV}$ [60]. Unlike the hadronic CR component, electrons, because of their low mass, suffer significant energy losses (pro- 


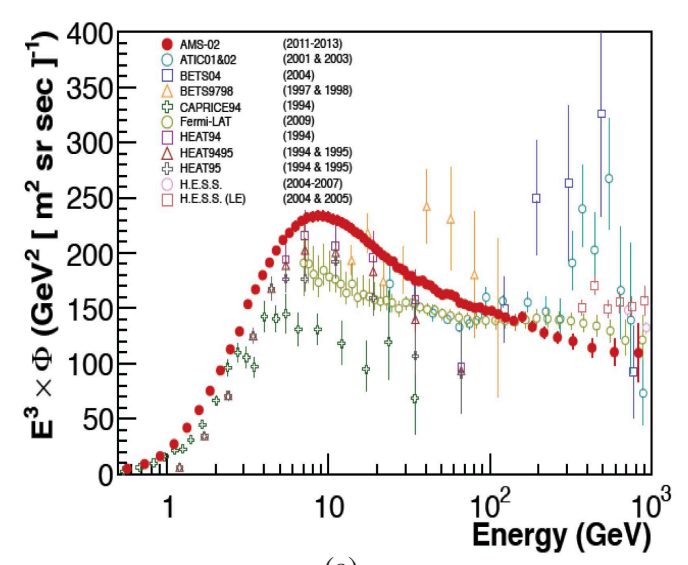

(a)

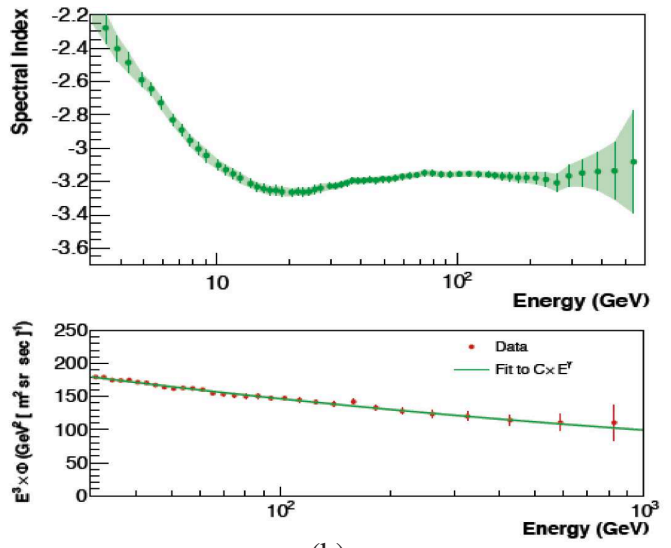

(b)

Figure 7: (a) The AMS $e^{+}+e^{-}$flux multiplied by $E^{3}$ as a function of energy $E$, compared with earlier results. (b) Top: spectral index of the all electron flux as a function of energy. Bottom panel: single powerlaw fit to the AMS $e^{+}+e^{-}$flux multiplied by $E^{3}$ above $30.2 \mathrm{GeV}$ [67].

portional to their squared energy) during propagation in the Galaxy through synchrotron radiation in the galactic magnetic field and inverse Compton scattering off CMB photons. These large energy losses explain the steeper electron spectrum compared to that of protons, and place an upper limit on the age $\left(\sim 10^{5} \mathrm{yr}\right)$ and distance $(<1 \mathrm{kpc})$ of the astrophysical sources of TeV electrons that can be observed at Earth. Since the number of such nearby SNRs is limited, the electron energy spectrum above $1 \mathrm{TeV}$ could exhibit spectral features and, at very high energies, a measurable anisotropy in the electron arrival directions is expected, due to the locality of the possible sources. Possible excess in the electron spectrum could also result from a primary source of positrons, which seems necessary to explain the rise in positron fraction with energy, recently observed by PAMELA [61] and AMS [62]. Infact such a source, either of astrophysical (e.g. pulsars) or exotic (e.g. dark matter) origin, would contribute with an equal number of positrons and electrons.

Measurements of the CR $e^{ \pm}$fluxes have been pursued since 1970's by balloon-borne and spacebased experiments. Because of the low intensity of the $e^{ \pm}$signals and the large CR proton background, the main requirements for these instruments are a large acceptance, long exposure time and an excellent $e / p$ discrimination capability. Calorimeters can be used to measure the "all" electron $\left(e^{+}+e^{-}\right)$spectrum, while separation between $e^{+}$and $e^{-}$makes mandatory the use of a magnetic spectrometer to determine the sign of the charge.

In recent years, a great interest has arisen from several new electron measurements. ATIC-2 reported an excess in the all electron spectrum at energies between 300 and $800 \mathrm{GeV}$ [63], which was not confirmed by subsequent measurements by Fermi and HESS. Fermi data follow a powerlaw spectrum with spectral index $-3.08 \pm 0.05$ [64], consistent with HESS result, which moreover reported a rapid steepening above $1 \mathrm{TeV}$ [65]. At this ICRC, AMS reported precision measurement of the fluxes of $\mathrm{CR} e^{-}, e^{+}$and $e^{+}+e^{-}[66]$. Unlike the $p$ and He analyses, the energy of electrons is measured with the ECAL, and the energy bins are chosen according to its energy resolution ( $\sim 2 \%$ at $300 \mathrm{GeV}$ ). Statistical estimators, based on Boosted Decision Tree and log-likelihood algorithms, have been developed to discriminate $e^{ \pm}$from $p$ by exploiting their different shower shapes in ECAL and energy deposited in TRD proportional tubes. The $p$ rejection power of ECAL is $\sim 10^{4}$ above few $\mathrm{GeV}$ for $90 \% e^{ \pm}$selection efficiency, while for TRD it reaches $10^{4}$ at $30 \mathrm{GeV}$ 
and decreases rapidly above. In each energy bin, signal and background yields are obtained with a data-driven template-fit approach. The $e^{+}+e^{-}$flux is not computed as the sum of $e^{-}$and $e^{+}$fluxes, but with a dedicated analysis that allowed to reduce the systematic uncertainties and increase the statistics of the sample at higher energies. In 30 months of operations, AMS identified $10.6 \times 10^{6}$ $e^{+}+e^{-}$events between $0.5 \mathrm{GeV}$ and $1 \mathrm{TeV}$. The resulting flux (Fig.7(a) is consistent with a single power-law above $30 \mathrm{GeV}$ with spectral index $\gamma=-3.170 \pm 0.008$ (stat+syst) \pm 0.008 (energy scale), as shown in Fig. 7(b)] No structures are observed in the $e^{+}+e^{-}$spectrum [67].

As far as the $e^{-}$spectrum is concerned, only in recent years $\mathrm{CR} e^{-}$above $50 \mathrm{GeV}$ were identified for the first time by PAMELA, which measured the $e^{-}$spectrum between 1 and $625 \mathrm{GeV}$. This is well described by a single power-law energy dependence with spectral index $-3.18 \pm 0.05$ above the energy of $30 \mathrm{GeV}$, and shows no significant spectral features within the errors [68].

A precise measurement of the $e^{-}$flux was presented by AMS (Fig. 8(a)], based on the identification of $9.23 \times 10^{6} e^{-}$events in the energy range $0.5-700 \mathrm{GeV}$ [66]. The AMS $e^{-}$spectrum cannot be fitted to a single power-law over the entire energy range not affected by solar modulation $(>10$ $\mathrm{GeV}$ ). It turned out that the spectral index $\gamma_{e^{-}}$has an energy dependence (Fig. $8(\mathrm{~d})$ ) and hardens at $\sim 30 \mathrm{GeV}$ [69]. Further discussion of this point invokes the comparison with the $e^{+}$flux behaviour and is presented in section 5.1 .

The multi-TeV region, where there is high potential for studying local cosmic accelerators, is still largely unexplored. Thanks to their large collection area, Imaging Atmospheric Cherenkov Telescopes (HESS, MAGIC) proved to be able to extend the $e^{+}+e^{-}$spectrum beyond $1 \mathrm{TeV}$ with large statistics, at the expense of a quite uncertain identification of the CR particle inducing the air shower. In [70], the ground-based experiment VERITAS reported a measurement of the electron spectrum between $300 \mathrm{GeV}$ and $4 \mathrm{TeV}$. The electron signal extraction is strongly based on Monte Carlo simulations and multivariate analysis methods, resulting in large systematic uncertainties on the energy scale, and significant contamination from gamma-ray events and protons. VERITAS data confirms a cutoff in the spectrum around $1 \mathrm{TeV}$, first observed by HESS [65], above which the spectral index rapidly changes from $\approx-3.2$ to $\approx-4.1$.

An interesting attempt to measure directly the electron flux at several $\mathrm{TeV}$ exploiting a novel detection technique was presented by the balloon-borne experiment CREST (Cosmic Ray Electron Synchrotron Telescope) [71]. Synchrotron X-rays emitted from $\mathrm{TeV}$ electrons bending in the geomagnetic field are detected by means of an array of $\mathrm{BaF}_{2}$ crystals readout by photomultipliers and surrounded by a plastic scintillator veto system. Electrons are identified by the characteristic signature of synchrotron photons, which form a co-linear, isochronous hit pattern in the detector. The effective acceptance of the instrument is very large $\left(18.5 \mathrm{~m}^{2} \mathrm{sr}\right.$ at $\left.5 \mathrm{TeV}\right)$, because only photons, and not directly electrons, are required to cross the detector. Moreover it increases with the electron energy, as the synchrotron photon yield. CREST was flown for 10 days over Antarctica in 2011. Unfortunately, the measurement was affected by the high level of photon background due to secondary production in the atmospheric overburden, which overwhelmed the expected synchrotron signals. Only a preliminary upper limit on the integral electron flux $\left(<7.11 \times 10^{-3} \mathrm{~m}^{-2} \mathrm{sr}^{-1} \mathrm{~s}^{-1}\right)$ was calculated at energies $>15 \mathrm{TeV}$.

Finally, on the low-energy side, it is worth recalling the Voyager 1 measurement of the unmodulated electron spectrum between 10 and $100 \mathrm{MeV}$ in the local ISM [34]. 


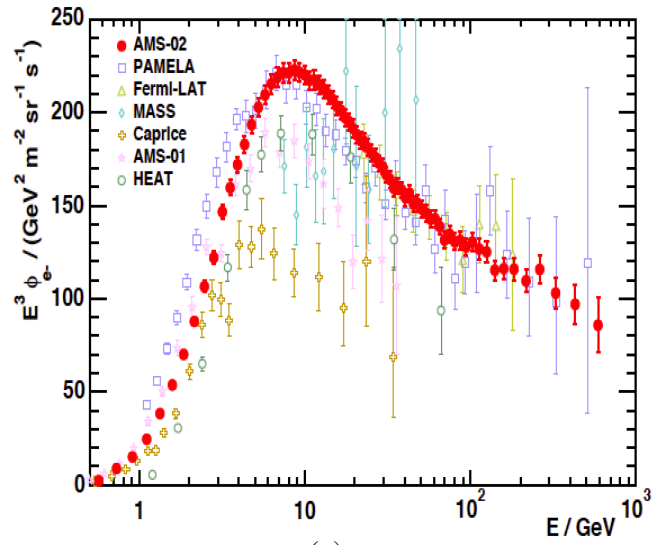

(a)

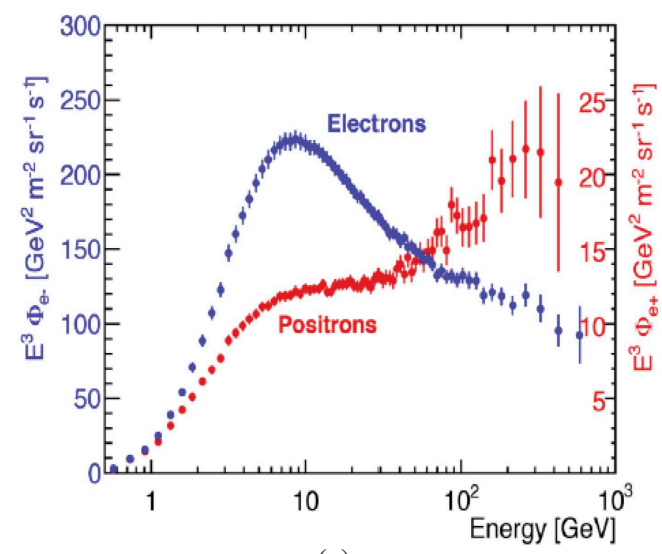

(c)

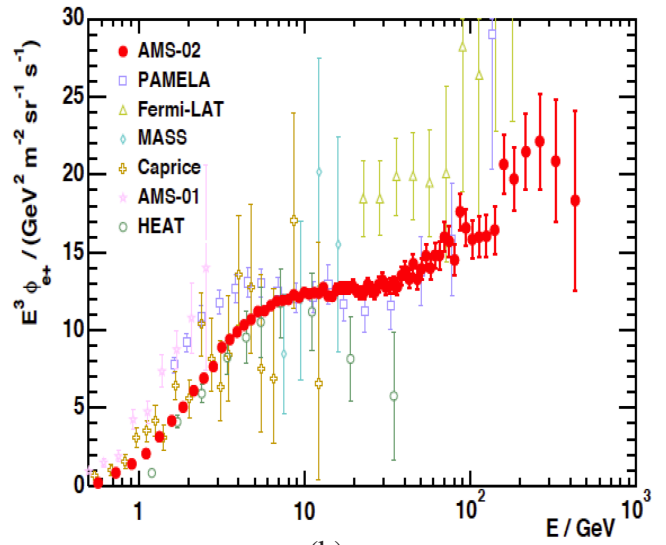

(b)

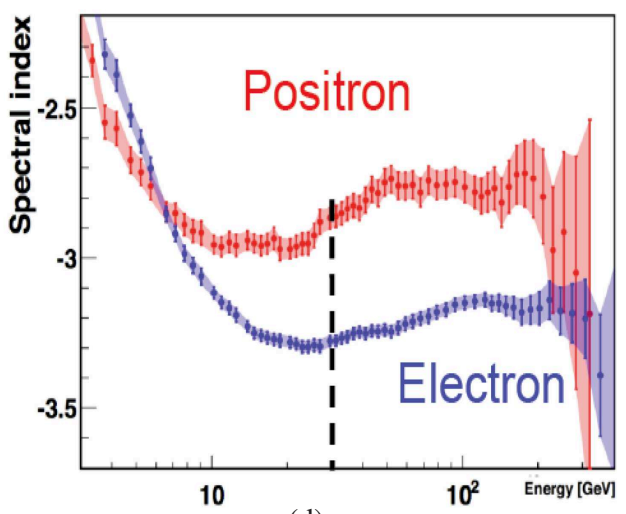

(d)

Figure 8: (a) $e^{-}$and (b) $e^{+}$flux multiplied by $E^{3}$ as measured by AMS, compared with earlier measurements. (c) Comparison of the $e^{ \pm}$fluxes measured by AMS. (d) The spectral indices $\gamma_{e^{ \pm}}$of the AMS $e^{ \pm}$ fluxes as a function of energy [66, 69].

\section{Antimatter}

Positrons and antiprotons are rare components of CRs. Since they are thought to be mainly of secondary origin, i.e. they are generated through collisions of CR nuclei with atoms of the ISM, detailed measurements of their energy spectra can provide important information about the CR propagation mechanisms. However the recent data from PAMELA and AMS show unexpected features in these spectra that require new interpretations.

\subsection{Positrons}

A major experimental challenge in the $e^{+}$measurement is represented by the need to reject the overwhelming background of protons $\left(p / e^{+}\right.$flux ratio $\sim 10^{3}$ at $\left.10 \mathrm{GeV}\right)$ and electrons $\left(e^{-} / e^{+} \sim 10\right.$ at $10 \mathrm{GeV}$ ). Discrepant results were reported by pioneering experiments, which were affected by a significant misidentification of the proton background. In the 1990's, the balloon-borne magnetic spectrometers HEAT [72] and CAPRICE [73], and AMS-01 in space [74] measured the positron fraction $e^{+} /\left(e^{+}+e^{-}\right)$up to $30 \mathrm{GeV}$, though with limited statistics above $10 \mathrm{GeV}$. A predominantly 


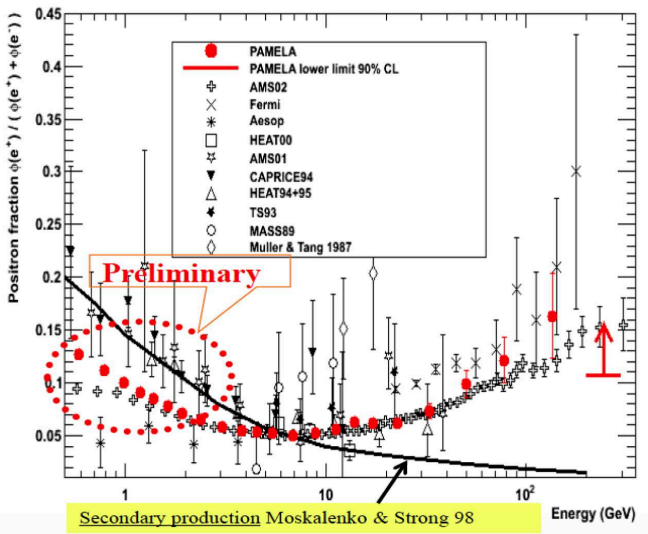

(a)

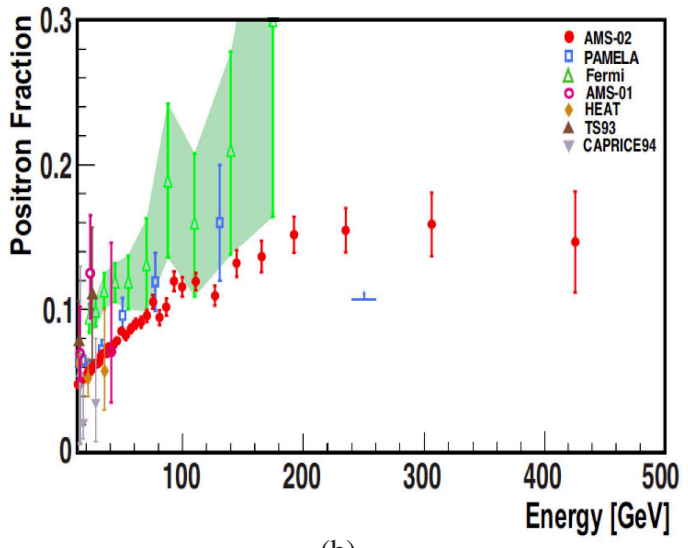

(b)

Figure 9: (a) The positron fraction in the energy range 0.5-200 GeV. Preliminary unpublished measurements by PAMELA in the low energy region $(<2 \mathrm{GeV})$ were presented at the ICRC [11]. The low-energy discrepancy between PAMELA and AMS with data collected during the 1990's is interpreted in terms of charge-sign solar modulation effects [77]. (b) The AMS positron fraction as a function of energy above 10 $\mathrm{GeV}$, where it begins to increase, compared with PAMELA and Fermi results [76].

decreasing positron fraction with increasing energy was observed with a small excess around 7 $\mathrm{GeV}$. The first measurement of $e^{+} /\left(e^{+}+e^{-}\right)$reaching $100 \mathrm{GeV}$ with significantly better precision than earlier data, was reported by PAMELA [61]. It clearly shows a rise of the positron fraction above $10 \mathrm{GeV}$ (Fig. 9(a)), in conflict with secondary production mechanisms, predicting a decresing trend with energy. Fermi LAT, though not equipped with a magnet, confirmed the positron rise between 20 and $200 \mathrm{GeV}$, with large systematic uncertainties, by exploiting the Earth's shadow to separate $e^{-}$from $e^{+}$[75].

In these ICRC papers [5, 66, 76], AMS presented its recently published results of the positron fraction and $e^{+}$flux. The ratio $e^{+} /\left(e^{+}+e^{-}\right)$was measured with unprecedented precision from 0.5 to $500 \mathrm{GeV}$ [62]. It steadily increases from 10 to $\sim 250 \mathrm{GeV}$ then reaching a maximum at $275 \pm 32$ $\mathrm{GeV}$ (Fig. 9(b)]. At higher energies, the fraction is no longer increasing but the data are still statistically limited to understand its behaviour. The observed rise in the positron fraction is due to an excess of positrons as pointed out by comparing the individual AMS $e^{-}$and $e^{+}$fluxes, shown in Fig. 8. Similarly to $e^{-}$flux, the $e^{+}$flux cannot be described by a single power-law over the entire energy range; its spectral index $\gamma_{e^{+}}$shows an energy dependence and hardens at $\sim 30 \mathrm{GeV}$. Moreover the spectral indices for $e^{+}$and $e^{-}$are significantly different with $\gamma_{e^{-}}<\gamma_{e^{+}}$between 20 $\mathrm{GeV}$ and $200 \mathrm{GeV}$ (Fig. 8(d), i.e. the electron flux is softer than the positron one. This demonstrates that the increase with energy observed in the positron fraction is due to the hardening of the positron spectrum and not to the softening of the electron spectrum above $10 \mathrm{GeV}$ [69].

The sharp rise observed in the positron fraction prompted many theoretical works trying to explain the excess of $e^{+}$, either including additional sources of high-energy $e^{ \pm}$or reassessing critically the secondary $e^{ \pm}$production from CR interactions with ISM. As far as this latter case is concerned, it was shown, for instance, that by tuning the CR propagation parameters [78], or with inhomogeneous diffusion models [19], is still possible to reproduce the data under the assumption of purely secondary production. In [79], the positron excess is explained taking into account $e^{ \pm}$produced as secondaries in the SNRs where CRs are accelerated. Conversely, astrophysical objects like nearby 
pulsars, or dark matter (DM) particles have been proposed as possible primary sources of positrons. In [80], both these hypotheses have been tested against the recent AMS data. They showed that leptophilic DM is disfavoured, while DM annihilation in quarks and gauge or Higgs bosons would be able to produce the positron excess, but only assuming high cross-sections not compatible with constraints from other observations. Instead an explanation based on a single pulsar seems more feasible, and five known nearby objects are identified which can fit the data. In [81], the interplay between astrophysical sources and DM is studied, showing that most of the primary $e^{+}$component can be produced by Pulsar Wind Nebulae (PWNe), with possible sub-leading components from SNRs and DM (Fig. 11(a)]. In [82], a better fit to the AMS $e^{+}+e^{-}$spectrum above $100 \mathrm{GeV}$ is obtained by adding a contribution from the pulsars Vela-X and Monogem to the standard local interstellar spectrum calculated with GALPROP.

However, the uncertainties on CR propagation parameters significantly affect the predicted positron rise for both the scenarios [83], thus strongly limiting at the moment the possibility to ascertain the origin of the excess. It was remarked that a different shape of the positron fraction above 500 $\mathrm{GeV}$ is expected depending on the astrophysical or DM origin of the $e^{+}$excess. That might be explored with future AMS data. Moreover in case of a few pulsar sources, a dipole anisotropy of order $1 \%$ should be observed at hundreds of GeV. So far, AMS measured a $e^{+} / e^{-}$ratio consistent with isotropy [62], while anisotropies searches separately for $e^{ \pm}$are underway [84]. A study of the arrival directions of $e^{+}$and $e^{-}$taking into account the effects of the Earth's geomagnetic field was presented by PAMELA. Results are consistent with isotropy at all angular scales considered. An upper limit of 0.076 at $95 \%$ confidence level on the amplitude of dipole anisotropy is calculated for positrons [85].

Several works based on analyses of PAMELA data at low energies $(<10 \mathrm{GeV})$ have been also presented at the ICRC, as for instance the study of the time variation of $e^{ \pm}$fluxes over the $23^{\text {rd }}$ solar minimum [86] and of the positron fraction betwen 2006 and 2015, aimed at investigating charge-sign dependent solar modulation effects; and the study of the secondary $e^{ \pm}$stably trapped and albedo components produced in interactions of CR protons with the residual atmosphere [87].

\subsection{Antiprotons}

The major difficulty in measuring the antiproton flux is the positive identification of the rare antimatter particles in the presence of a large background, mostly consisting of electrons and, for experiments performed with balloons at small atmospheric depths, secondary pions and muons, which are both produced in the interaction with the atmosphere. Early measurements of antiprotons were severely compromised by large background contamination due to an inadequate particle identification. Magnetic spectrometers in 1990's measured the antiproton flux below $10 \mathrm{GeV}$ and only CAPRICE98 [88] reached few tens of GeV. BESS95+97 showed that the $\bar{p}$ spectrum has a distinct peak at $2 \mathrm{GeV}$ with the flux decreasing at lower energies, as expected from the kinematic constraints of $\bar{p}$ production in the ISM [89]. That confirmed that the secondary component is dominant in the $\mathrm{CR} \bar{p}$ flux. However a possible excess at energies lower than the secondary peak, which was not ruled out by BESS95+97 data, could result from cosmologically primary sources of $\bar{p}$, like evaporation of primordial black holes (PBH). This exotic component was later excluded by BESS-Polar II precise measurement between 0.17 and $3.56 \mathrm{GeV}$ near solar minimum in Dec. 2007 [90]. The first observations of both $\bar{p}$ flux (Fig. 10(a)] and $\bar{p} / p$ flux ratio extending to high 


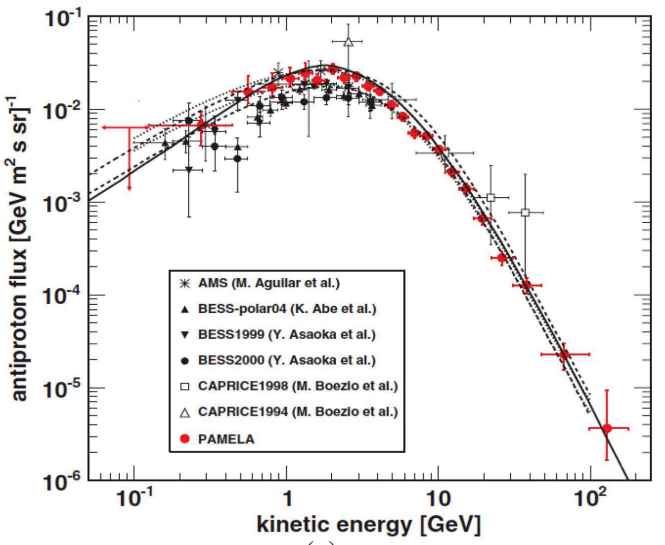

(a)

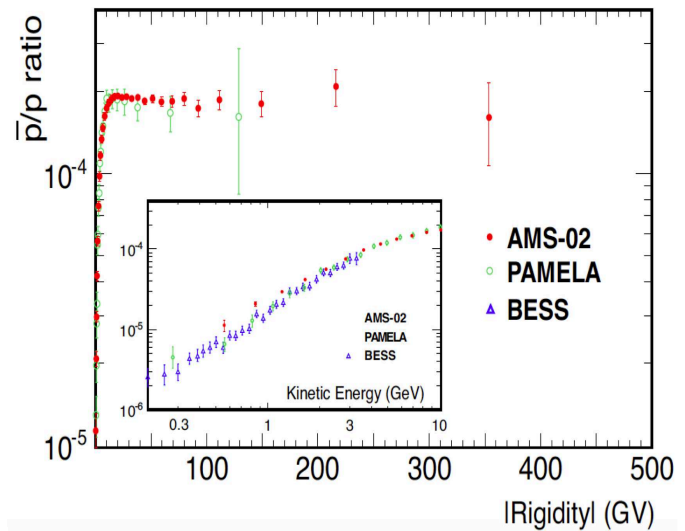

(b)

Figure 10: (a) $\bar{p}$ flux measured by PAMELA, compared with previous results and theoretical calculations for a pure secondary $\bar{p}$ production [91]. (b) AMS $\bar{p} / p$ ratio up to $450 \mathrm{GV}$ [76], compared with PAMELA [91] and BESS-Polar [90] results. An expanded view of the low-energy region is shown in the inset plot.

energy $(180 \mathrm{GeV})$ were performed by PAMELA [91]. At the ICRC, AMS presented a preliminary measurement of the $\bar{p} / p$ ratio, based on the identification of $2.9 \times 10^{5} \bar{p}$ selected in the rigidity range $1-450 \mathrm{GV}$ [76]. This result has unprecedented precision and clearly shows that the $\bar{p} / p$ ratio is almost constant with rigidity above $100 \mathrm{GV}$ (Fig. 10(b)].

This unexpected behaviour at high energy has triggered speculations about a possible primary antiproton component originating from annihilation of DM particles. At the beginning, a misleading comparison of AMS data with old secondary production predictions (e.g. [92]), which indicated a rapid decrease of the $\bar{p} / p$ ratio with increasing energy, seemed to point to the DM scenario. Hence in order to clarify the origin of the anomaly, calculations of the secondary $\bar{p} / p$ flux ratio have been reassessed using updated propagation models, taking into account additional effects previously neglected (e.g. $\bar{p}$ energy losses (including tertiary component), diffusive reacceleration, solar

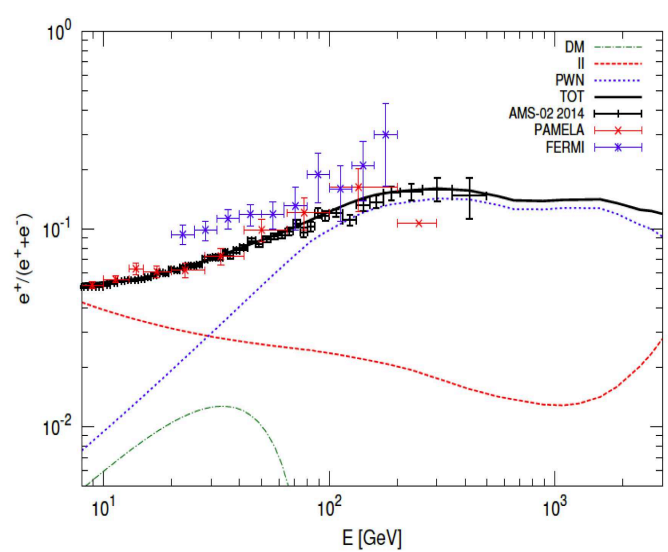

(a)

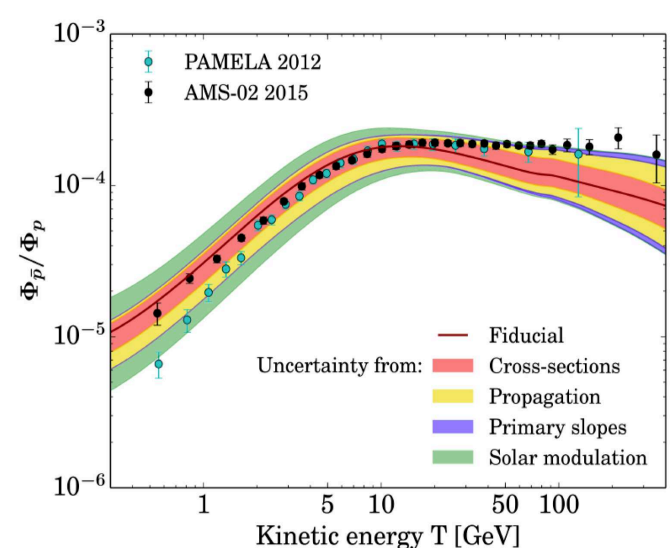

(b)

Figure 11: (a) Prediction for the positron fraction calculated in [81], including contributions from secondaries, PWNe and DM annihilating into the $\mu^{+} \mu^{-}$channel. (b) The combined total uncertainty on the secondary $\bar{p} / p$ ratio calculated in [94], superimposed to PAMELA and AMS data. 
modulation), harder $p$ and He spectra at high energy, diffusion coefficient consistent with recent $\mathrm{B} / \mathrm{C}$ data, newer data on $\bar{p}$ production cross sections. Several theoretical works have been presented driven by this approach and aimed at a critical evaluation of the relevant uncertainties affecting the computations [93-97]. An example in shown in Fig. 11(b), They all come to a similar conclusion: that the flat $\bar{p} / p$ ratio at high energy is consistent with pure secondary production within the error band of the updated predictions. However, given the current uncertainties, mainly on propagation parameters, a possible $\bar{p}$ contribution from DM cannot be ruled out. More accurate $\bar{p}$ data at high energy, expected from AMS in next years, combined with an improved modeling of the secondary production, will allow to search with better sensitivity for possible sub-dominant DM signals in a large background of secondary $\bar{p}$.

\section{Future experiments}

Several new experiments have been presented at the ICRC. Some of them are already operating 2 (NUCLEON, CALET) or foreseen to start taking data soon (DAMPE, ISS-CREAM, CSES), others have only been proposed (HNX, HELIX, GAPS) or they are in an initial phase of design and development (GAMMA-400). Some experiments address broad observation targets (CALET, NUCLEON, DAMPE, ISS-CREAM), while others are more focused on specific measurements (CSES, HNX, HELIX, GAPS). It can be noticed that the projects that are expected to produce results in the next few years (CALET, NUCLEON, ISS-CREAM and DAMPE) are all based on calorimetric instruments, featuring large depth and acceptance and long exposure aimed at measuring the individual spectra of CR nuclei up to hundreds of TeV approaching the knee, and the all electron spectrum up to several $\mathrm{TeV}$. Then they are foreseen to provide overlaps with AMS $p, \mathrm{He}$, and $e^{+}+e^{-}$spectra and extend such measurements well beyond the energy limit imposed by the MDR of the spectrometer. In the following paragraphs, I will describe the main experiments which will likely present new results at the next ICRC, comparing their detection techniques and performance, observation targets and expected outcome. Finally, the most interesting proposals presented at the ICRC will be briefly sketched.

\subsection{NUCLEON}

NUCLEON is a russian spaced-based detector aimed at measuring the energy spectra of individual $\mathrm{CR}$ nuclei from $Z=1$ to $Z=30$ in the energy range from $100 \mathrm{GeV}$ up to $1 \mathrm{PeV}$. It was successfully launched on a RESURS-P satellite on Dec. $26^{\text {th }} 2014$ and it is planned to take data for 5 years. The instrument includes four silicon pad detectors to measure the particles charge, a carbon target, six scintillator layers for the trigger system, a tracker made of six layers of Si microstrips interleaved with thin W layers, and a Si microstrip-Tungsten sampling calorimeter. The total thickness is about 16 radiation lengths $\left(X_{0}\right)$. The energy of CR particles is determined by two independent techniques: a Kinematic Lightweight Energy Method (KLEM), based on the measurements of the spatial density of secondary shower particles produced in the carbon target and $\mathrm{W}$ layers by means of the Si microstrip tracker, and a traditional ionization calorimeter technique. The sampling calorimeter can measure also electrons in the energy range $100 \mathrm{GeV}-3 \mathrm{TeV}$. The effective geometric factor is more than $0.2 \mathrm{~m}^{2} \mathrm{sr}$ for nuclei and $0.06 \mathrm{~m}^{2} \mathrm{sr}$ for electrons [98].

\footnotetext{
2 at the time of writing
} 


\subsection{CALET (CALorimetric Electron Telescope)}

CALET is a space mission led by the Japanese Space Agency (JAXA) with the participation of the Italian Space Agency (ASI) and NASA. CALET was launched on August 19 ${ }^{\text {th }}, 2015$ by the Japanese rocket H-II Transfer Vehicle (HTV-5) and robotically installed on the Japanese Experiment Module Exposure Facility (JEM-EF) of the ISS on August $24^{\text {th }}$, for a two-year mission, with a possible first extension to 5 years [99]. Its main scientific goal is to search for possible clues of the presence of astrophysical sources of high-energy electrons nearby the Earth or signatures of $\mathrm{DM}$, by measuring accurately the $e^{-}+e^{+}$spectrum from $1 \mathrm{GeV}$ up to several $\mathrm{TeV}$ [100]. CALET will also measure the energy spectra and elemental composition of $\mathrm{CR}$ nuclei from $\mathrm{H}$ to $\mathrm{Fe}$ up to hundreds of $\mathrm{TeV}$, and the abundance of UHGCRs at few GeV/amu up to about $\mathrm{Z}=40$ [101]. The instrument consists of two layers of segmented plastic scintillators to identify the particle charge, a thin tungsten-scintillating fiber imaging calorimeter providing accurate particle tracking and identification by multiple $\mathrm{dE} / \mathrm{dx}$ sampling, and a thick PWO crystal calorimeter to measure the energy of $\mathrm{CRs}$ with excellent resolution and electron/hadron separation up to the multi-TeV scale. The total thickness is equivalent to $30 X_{0}$ and 1.3 proton interaction lengths $\left(\lambda_{I}\right)$. The geometrical factor is $0.12 \mathrm{~m}^{2} \mathrm{sr}$ and the total weight is $613 \mathrm{~kg}$. An extensive campaign of beam tests for calibration was carried out at GSI and CERN-SPS from 2011 to 2015 with beams of accelerated electrons, muons, protons and ion fragments [102]. The main features of the detector are: a charge resolution ranging from 0.15 for B to $0.30-0.35$ charge units in the Fe region; an energy resolution better than $2 \%$ for electrons above $100 \mathrm{GeV}$ [103]; an angular resolution of $0.1^{\circ}$ for electrons and better than $0.5^{\circ}$ for hadrons [104]; a good energy linearity for electrons and hadrons up to several $\mathrm{TeV}$ [105]; a proton rejection power $\sim 10^{5}$ with $80 \%$ electron efficiency [106].

\subsection{ISS-CREAM (Cosmic Ray Energetics And Mass)}

ISS-CREAM have been built transforming the CREAM payload, successfully flown in six flights over Antarctica, for accommodation on the ISS JEM-EF for a 3 year mission [107]. The exposure will be increased by an order of magnitude allowing ISS-CREAM to extend the measurement of the energy spectra of CR nuclei up to hundreds of TeV. The ISS-CREAM payload $(\sim 1300$ $\mathrm{kg}$ ) is currently being integrated at NASA Wallops Flight Facility and scheduled to be launched mid of 2016 by SpaceX. The detector includes: four layers of Si pixels to measure redundantly the particle charge with resolution of 0.2 charge units; a $0.5 \lambda_{I}$-thick carbon target to induce the inelastic interaction of the incoming nuclei; a sampling calorimeter made of 20 layers of alternating tungsten plates and scintillating fibers, providing energy measurement, particle tracking and trigger; top and bottom plastic scintillator counters and a boronated scintillator detector for $e / p$ separation. While the Si charge detector and the calorimeter were already used in the balloon campaign and rearranged for ISS-CREAM payload, the last two detectors have been newly developed for the space mission, in order to add sensitivity also to CR electrons.

\subsection{DAMPE (DArk Matter Particle Explorer)}

DAMPE is a satellite experiment promoted by the Chinese Academy of Sciences and built in collaboration with institutions from Italy and Switzerland [108]. The launch is planned on Dec. 2015. The detector consists of four subsystems: plastic scintillator strips which serves as 
anti-coincidence detector for photon identification, as well as charge detector for CRs; a silicontungsten tracker-converter (STK) providing track reconstruction, photon detection, and CR charge measurement; the BGO imaging calorimeter to measure the incoming particle energy; and the neutron detector made of boron-doped plastic plates to detect delayed neutron emerging from hadron showers in order to improve the $e / p$ discrimination. DAMPE is designed to measure electrons and photons between $5 \mathrm{GeV}$ to $10 \mathrm{TeV}$ with excellent resolution $(1.5 \%$ at $100 \mathrm{GeV})$ to search for possible DM signatures, and CR nuclei between $10 \mathrm{GeV}$ and $100 \mathrm{TeV}$ with individual element separation. The total thickness of the calorimeter is equivalent to $31 X_{0}$ and $1.6 \lambda_{I}$. The geometrical acceptance is $\sim 0.3 \mathrm{~m}^{2} \mathrm{sr}$ for $e^{-}$and $\gamma$, and $\sim 0.2 \mathrm{~m}^{2} \mathrm{sr}$ for hadrons.

\subsection{CSES (China Seismo-Electromagnetic Satellite)}

CSES is a chinese-italian space mission scheduled to be launched in 2016. It aimed at studying the electromagnetic anomalies from Earth and ionosphere perturbations possibly associated with earthquakes, in order to develop new technologies to prevent disaster. In particular the HEPD (High Energy Particle Detector, developed by the italian group) onboard the satellite will search for correlations between the precipitation of low energy electrons trapped within the Van Allen Belts and earthquakes with Richter magnitude $>5$. Besides this scope, HEPD will study the low-energy component of CRs of solar and galactic origin, complementing PAMELA and AMS measurements in the sub-GeV region. It consists of two layers of plastic scintillators for trigger, two planes of double-side Si microstrip detectors to measure the incident particle direction, a calorimeter made of plastic and LYSO crystal scintillators, and a veto system on the sides. The total weight is 35 $\mathrm{kg}$ and the mechanical size roughly $20 \times 20 \times 40 \mathrm{~cm}^{3}$. HEPD can measure electrons from 1 to 200 $\mathrm{MeV}$, and protons from 30 to $200 \mathrm{MeV}$ with very good separation, an energy resolution $<10 \%$ at 5 $\mathrm{MeV}$ and an angular resolution of $\sim 8^{\circ}[109]$.

\subsection{Proposed experiments}

GAMMA-400 is a next generation gamma-ray space observatory planned for 2023-2025 [110]. Though specifically designed to measure gamma-ray sources with unprecedented accuracy, the instrument also includes an innovative homogeneous and isotropic calorimeter made of cubic crystals, featuring a depth of $54 \mathrm{X}_{0}$ or $2.5 \lambda_{I}$ when detecting laterally incident particles. The resulting improved shower containment and the large acceptance (a few $\mathrm{m}^{2} \mathrm{sr}$ ) would allow to measure $\mathrm{CR}$ nuclei up to the "knee" with very good energy resolution.

Heavy Nuclei eXplorer (HNX) is a NASA experiment conceived as the successor of SuperTIGER in space [111]. It exploits complementary active detectors (silicon strips and Cherenkov counters with acrylic and silica-aerogel radiators), and passive glass tiles to achieve $50 \mathrm{~m}^{2} \mathrm{sr}$ geometric factor, required to measure nuclei up to the Actinides (Th, U, Pu). These are very important CR clocks, which can provide information about the absolute age of ultra-heavy GCRs since nucleosynthesis. HNX is designed to be accomodated in the DragonLab Capsule for a 2 year mission.

HELIX (High Energy Light Isotope eXperiment) is a balloon-borne experiment consisting of a superconducting magnet spectrometer, a Ring Imaging Cerenkov Detector and a Time-of-Flight system optimized to achieve a separation of adjacent light isotopes with 0.25 amu resolution [112]. The main goal is to measure with good statistics the ${ }^{10} \mathrm{Be} /{ }^{9} \mathrm{Be}$ abundance ratio up to $10 \mathrm{GeV} / \mathrm{n}$, 
where available data are lacking. This ratio is extremely important to provide information on the mean containment lifetime of CRs in the Galaxy and the diffusion coefficient.

The General Antiparticle Spectrometer (GAPS) is a large-acceptance balloon-borne experiment devoted to indirect DM search by measuring low-energy $(<1 \mathrm{GeV} / \mathrm{n}) \mathrm{CR}$ antideuterons and $\bar{p}$ with a new detection technique, nearly background free [113]. Antimatter particles are identified by the characteristic X-rays emitted by exotic atoms they form when they are stopped in a stack of $\mathrm{Si}(\mathrm{Li})$ detectors, and by the production of a $\pi$ or $p$ star when subsequently they annihilate.

\section{Conclusions}

A wealth of new direct CR measurements have been presented at the $34^{\text {th }}$ ICRC. Among many interesting results, I would like to recall the following:

- AMS precise measurements of the spectral hardening of $p$ and He spectra at $~ 300 \mathrm{GV}$, with He harder than $p$;

- preliminary AMS observation of a hardening also in Li spectrum, not in C one;

- new AMS B/C data, approaching TeV/n scale with unprecedented precision;

- Voyager-1 measurements of the unmodulated energy spectra of CR nuclei in the LISM;

- new SuperTIGER UHGCR data, pointing to OB associations as CR acceleration site;

- first detection of a primary CR clock $\left({ }^{60} \mathrm{Fe}\right)$ from CRIS;

- $e^{ \pm}$spectra inconsistent with a single power-law behaviour, with $e^{+}$harder than $e^{-}$;

- flat behaviour of AMS $\bar{p} / p$ ratio at high energy.

A great theoretical effort is currently underway to explain and interpret these new results, which challenge the standard paradigm of CR acceleration and propagation. A lot of new precise data are foreseen in the years to come from AMS and several new projects at the horizon.

I would like to thank the organizers of the conference for inviting me as rapporteur and the local organising committee for the support in collecting and making available talks and papers. I also thank Dr. M. Boezio, Prof. M.H. Israel, Prof. P.S. Marrocchesi, Dr. J.W. Mitchell, and Dr. N. Mori for useful comments during the preparation of the talk and paper.

\section{References}

[1] A.V. Apanasenko et al., Astropart. Phys.16 (2001) 13

[2] K. Asakimori et al., ApJ 502 (1998) 278

[3] Y.S. Yoon et al., ApJ 728 (2011) 122

[4] O. Adriani et al., Science 332 (2011) 69

[5] S. Ting, Pos(ICRC2015) 036

[6] V. Choutko, Pos(ICRC2015) 260

[7] M. Aguilar et al.,PRL 114 (2015) 171103

[8] S. Haino, Pos(ICRC2015) 236

[9] K. Sakai et al., Pos(ICRC2015) 436 
[10] A.D. Panov et al., Bull. Russ. Acad. Sci. Phys. 73 (2009) 564

[11] M. Boezio, Pos(ICRC2015) 037

[12] A. Obermeier et al., Pos(ICRC2015) 286

[13] P. Serpico, Pos(ICRC2015) 009

[14] Y.M. Butt, A.M. Bykov, ApJ 677 (2008) L21

[15] E. Parizot et al., A\&A 424 (2004) 747

[16] S. Thoudam, J. R. Hörandel, A\&A 567 (2014) A33

[17] D.C. Ellison, E.G. Berezhko, M.G. Baring, ApJ 540 (2000) 292

[18] V. Ptuskin, V. Zirakashvili, E.S. Seo, ApJ 763 (2013) 47

[19] N. Tomassetti, Pos(ICRC2015) 551

[20] L. Nava, Pos(ICRC2015) 541

[21] P. Blasi, E. Amato, P.D. Serpico, PRL 109 (2012) 061101

[22] S. Thoudam, J. R. Hörandel, MNRAS 435 (2013) 2532-2542

[23] W. Liu, P. Salati, X. Chen, Res. Astron. Astrophys. 15 (2015) 1

[24] Y. Zhang et al., Pos(ICRC2015) 546

[25] N. Tomassetti, Pos(ICRC2015) 552

[26] V.I. Zatsepin, N.V. Sokolskaya, A\&A 458 (2006) 1

[27] M.A. Malkov et al., PRL 108 (2012) 081104

[28] L.O. Drury, MNRAS 415 (2011) 1807

[29] Y. Ohira, K. Ioka, ApJ 729 (2011) L13

[30] P. Blasi, E. Amato, JCAP 1201 (2012) 011

[31] C. Consolandi et al., Pos(ICRC2015) 117

[32] A. Bruno, Pos(ICRC2015) 288

[33] E.C. Stone, Pos(ICRC2015) 026

[34] A.C. Cummings et al., Pos(ICRC2015) 318

[35] N. Picot-Clémente et al., Pos(ICRC2015) 425

[36] O. Adriani et al., ApJ 770 (2013) 2

[37] W. Menn et al., Pos(ICRC2015) 270

[38] H.S. Ahn et al., ApJ 707 (2010) 593

[39] M. Ave et al., ApJ 678 (2008) 262

[40] H.S. Ahn et al., ApJ 714 (2010) L89-L93

[41] L. Derome, Pos(ICRC2015) 303

[42] M. Heil, Pos(ICRC2015) 289

[43] E. Vannuccini et al., Pos(ICRC2015) 380 
[44] W. Menn et al., Pos(ICRC2015) 269

[45] H.S. Ahn et al., Astropart. Phys. 30 (2008) 133

[46] A. Obermeier et al., ApJ 752 (2012) 69

[47] A.D. Panov et al., Proc. of the $23^{\text {rd }}$ ICRC, Merida, vol. 2 (2008) 3-6

[48] A. Oliva, Pos(ICRC2015) 265

[49] Y. Genolini, A. Putze, P. Salati, P.D. Serpico, Pos(ICRC2015) 539

[50] S. Kunz, I. Gebauer, Pos(ICRC2015) 542

[51] R. Aloisio, P. Blasi, P. Serpico, submitted to A\&A, arXiv:1507.00594

[52] B.F. Rauch et al., ApJ 697 (2009) 2083

[53] W.R. Binns et al., ApJ 634 (2005) 351

[54] T. Hams et al., Pos(ICRC2015) 038

[55] T. Hams et al., Pos(ICRC2015) 435

[56] R.P. Murphy et al., Pos(ICRC2015) 438

[57] A.W. Labrador et al., Pos(ICRC2015) 341

[58] M.H. Israel et al., Pos(ICRC2015) 275

[59] M.E. Wiedenbeck et al., ApJ 523 (1999) L61

[60] K. Koyama et al., Nature 378 (1995) 255; F. Aharonian et al., Nature 432 (2004) 75

[61] O. Adriani et al., Nature 458 (2009) 607

[62] L. Accardo et al., PRL 113 (2014) 121101; M. Aguilar et al., PRL 110 (2013) 141102

[63] J. Chang et al., Nature 456 (2008) 362

[64] M. Ackermann et al., PRD 82 (2010) 092004

[65] F. Aharonian et al., A\&A 508 (2009) 561

[66] M. Duranti, Pos(ICRC2015) 273

[67] M. Aguilar et al.,PRL 113 (2014) 221102

[68] O. Adriani et al., PRL 106 (2011) 201101

[69] M. Aguilar et al.,PRL 113 (2014) 121102

[70] D. Staszak, Pos(ICRC2015) 411

[71] J. Musser et al., Pos(ICRC2015) 415

[72] S.W. Barwick et al., ApJ 482 (1997) L191

[73] M. Boezio et al., ApJ 532 (2000) 653

[74] M. Aguilar et al., Physics Letters B 646 (2007) 145

[75] M. Ackermann et al., PRL 108 (2012) 011103

[76] A. Kounine, Pos(ICRC2015) 300

[77] O. Adriani et al., PRL 111 (2013) 081102 
[78] K. Blum, B. Katz, E. Waxman, PRL 111 (2013) 211101

[79] P. Blasi, PRL 103 (2009) 051104

[80] M. Boudaud et al., Pos(ICRC2015) 1183

[81] M. Di Mauro, A. Vittino, Pos(ICRC2015) 1177

[82] D. Rozza et al., Pos(ICRC2015) 501

[83] M. Boudaud et al., Pos(ICRC2015) 540

[84] I. Gebauer et al., Pos(ICRC2015) 404 and 408

[85] B. Panico et al., Pos(ICRC2015) 410

[86] R. Munini et al., Pos(ICRC2015) 071

[87] V. Mikhailov, E. Mocchiutti, Pos(ICRC2015) 243 and 383

[88] D. Bergström et al. ApJ 534 (2000) L177

[89] S. Orito et al., PRL 84 (2000) 1078

[90] K. Abe et al., PRL 108 (2012) 051102

[91] O. Adriani et al., PRL 105 (2010) 121101

[92] F. Donato et al., PRL 102 (2009) 071301

[93] M. di Mauro et al., PRD 90 (2014) 085017

[94] G. Giesen et al., Pos(ICRC2015) 1184

[95] R. Cowsik, T. Madziwa-Nussinov, Pos(ICRC2015) 548

[96] R. Kappl, A. Reinert, M.W. Winkler, arXiv:1506.04145

[97] C. Evoli, D. Gaggero, D. Grasso, arXiv:1504.05175

[98] D. Podorozhny et al., Pos(ICRC2015) 295

[99] S. Torii, Pos(ICRC2015) 581

[100] H. Motz, Pos(ICRC2015) 1194

[101] B.F. Rauch, Pos(ICRC2015) 627

[102] T. Tamura, Pos(ICRC2015) 589

[103] G. Bigongiari, Pos(ICRC2015) 592

[104] P. Brogi et al., Pos(ICRC2015) 595

[105] Y. Akaike, Pos(ICRC2015) 613

[106] F. Palma, Pos(ICRC2015) 1196

[107] E.S. Seo, Pos(ICRC2015) 574

[108] X. Wu, Pos(ICRC2015) 1192

[109] R. Sparvoli, Pos(ICRC2015) 567

[110] N.P. Topchiev et al., Pos(ICRC2015) 1026

[111] J.W. Mitchell and M. Sasaki, Pos(ICRC2015) 681

[112] S.P. Wakely et al., Pos(ICRC2015) 682

[113] P. von Doetinchem et al., Pos(ICRC2015) 1219 